\title{
Limit Theorems of Occupation Times for Markov Processes
}

\author{
By
}

\author{
Yuji KasAHARA*)
}

\section{§0. Introduction}

Let $X_{t}$ be a temporally homogeneous Markov process with values in an abstract space and $f(x)$ be a measurable function on this space. The limiting distribution of random variable

$$
\frac{1}{u(t)} \int_{0}^{t} f\left(X_{s}\right) d s \quad \text { as } t \longrightarrow \infty
$$

where $u(t)$ is some normalizing function, has been investigated by many authors. A most general limit theorem was obtained by Darling and Kac [1], who also showed that, under suitable condition, the limit distribution must be Mittag-Leffler distribution. However, they confined themselves to the case where $f(x)$ is nonnegative. C. Stone [5] derived a limit theorem for processes including 1-dimensional diffusion processes with the infinitesimal generator $\frac{d}{d m} \frac{d}{d x}$. It is not assumed that $f(x)$ is nonnegative, but it is essential that $f(x)$ is not null-charged; $\int f(x) m(d x)$ $\neq 0$.

In this paper we study the case where $f(x)$ is null-charged. If $X_{t}$ is positively recurrent, the problem above can be reduced to the central limit theorem (see Tanaka [6]). A similar problem was treated by Dobrusin [2], who studied limit theorems for the 1-dimensional simple random walk.

The aim of this paper is to give a limit theorem for most general processes. Contrary to the case of [1], the limiting distribution is

Communicated by K. Itô, July 30, 1976.

*) Department of Mathematics, Kyoto University, Kyoto. 
bilateral Mittag-Leffler distribution (for the definition, see Appendix). We also prove that, under suitable conditions, the limiting distribution must be bilateral Mittag-Leffler distribution.

To prove these theorems, we use the method of Darling and Kac; we calculate the Laplace transform of the moments of $\int_{0}^{t} f\left(X_{s}\right) d s$ and appeal to Tauberian theorem. However, matters are more complicated since $f(x)$ may take negative values in our case.

In section 1, we will state our theorems in a general form and we give, as an example, a limit theorem for 1-dimensional Brownian motion. Sections 2 and 3 are devoted to the proof of the theorems in section 1. In the last two sections, we apply the main theorems to symmetric stable processes and 1-dimensional diffusion processes.

\section{§1. Main Theorems}

Let $X=\left(X_{t}, P_{x}\right)$ be a temporally homogeneous Markov process with state space $(E, \mathscr{B})$, where $E$ is a locally compact Hausdorff space and $\mathscr{B}$ the Borel $\sigma$-field of $E$. We assume there is a Radon measure $v(d x)$ such that the transition probability $p(t, x, d y)$ is absolutely continuous with respect to $v(d y)$;

$$
p(t, x, d y)=p(t, x, y) v(d y) .
$$

Let us denote by $G_{s}(s>0)$ the Green operator of $X$;

$$
G_{s} f(x)=\int_{E} G_{s}(x, y) f(y) v(d y)
$$

for any bounded measurable function $f$, where

$$
G_{s}(x, y)=\int_{0}^{\infty} e^{-s t} p(t, x, y) d t
$$

In the sequel, we assume that $G_{s}(x, y)$ has following representation;

$$
G_{s}(x, y)=h(s)+u(x, y)+\varepsilon(x, y ; s) .
$$




\section{[Assumptions]}

(A) $f(x)$ is a bounded measurable function on $E$ such that $\sup _{x \in E}\left|G_{s} f(x)\right|$ is bounded as $s \rightarrow 0$.

(B) $\lim _{s \rightarrow 0} h(s)=\infty$.

(C) $C=\iint u(x, y) f(x) f(y) v(d x) v(d y) \neq 0$.

(D) There exists a measurable function $1 \leqq \rho(x)<\infty$ satisfying the following;

$$
\begin{gathered}
\int|f(y)| \rho(y) v(d y)<\infty . \\
\frac{1}{\rho(x)} \int|u(x, y) f(y)| \rho(y) v(d y)
\end{gathered}
$$

is bounded in $x$.

$$
\begin{aligned}
& \frac{1}{\rho(x)} \int|\varepsilon(x, y ; s) f(y)| \rho(y) v(d y) \\
& \text { converges to } 0 \text { uniformly in } x \text { as } s \rightarrow 0 .
\end{aligned}
$$

Suppose (A) (D) are satisfied. Then we easily see that $f(x)$ satisfies the following condition;

$$
\int f(x) v(d x)=0
$$

This condition plays an essential part in the sequel. The condition (B) is, roughly speaking, equivalent to the recurrence of $X_{t}$, and in many cases, (C) is satisfied if $f(x) \neq 0 v$-a.e.. (D) is a rather technical assumption, and in a special case such as $f(x)$ has compact support, (D) can be replaced by a more natural assumption $\left(\mathrm{D}^{\prime}\right)$;

(D') (D.1') $\int|f(x)| v(d x)<\infty$.

(D.2') $u(x, y)$ is locally integrable and $\int|u(x, y) f(y)| v(d y)$ is bounded on $\{\xi ; f(\xi) \neq 0\}$.

(D.3') $\lim _{s \rightarrow 0} \int|\varepsilon(x, y ; s) f(y)| v(d y)=0$, and the convergence is uniform 
on $\{\xi ; f(\xi) \neq 0\}$.

Theorem 1. If $X$ and $f(x)$ satisfy $(\mathrm{A}) \sim(\mathrm{D})$ (or $\left.\left(\mathrm{D}^{\prime}\right)\right)$, and if $h(s)=s^{-\alpha} L(1 / s)$ with $L(1 / s)$ slowly varying as $s \rightarrow 0$, then, $0 \leqq \alpha \leqq 1, C>0$ and for each $x \in E$,

$$
\lim _{t \rightarrow \infty} P_{x}\left\{\frac{1}{\sqrt{C h(1 / t)}} \int_{0}^{t} f\left(X_{s}\right) d s<u\right\}=\tilde{g}_{\alpha / 2}(u), u \in \mathbb{R}^{1}
$$

where $\tilde{g}_{\alpha / 2}(u)=\frac{1}{\pi \alpha} \int_{-\infty}^{u} \sum_{j=1}^{\infty} \frac{(-1)^{j-1}}{j !} \sin \frac{\pi \alpha}{2} j \Gamma\left(\frac{\alpha}{2} j+1\right)|y|^{j-1} d y$.

Theorem 1 has the following converse;

Theorem 2. If $X$ and $f(x)$ satisfy (A) (D) (or $\left.\left(\mathrm{D}^{\prime}\right)\right)$, and if there is a nondegenerate distribution function $G(x)$ such that

$$
\lim _{t \rightarrow \infty} P_{x}\left\{\frac{1}{u(t)} \int_{0}^{t} f\left(X_{s}\right) d s<u\right\}=G(u), \text { a.e. } u
$$

holds for some $x \in E$ and for some appropriate nondecreasing function $u(t) \nearrow \infty$, then $h(s)=s^{-\alpha} L(1 / s)$ for some $\alpha(0 \leqq \alpha \leqq 1)$ and slowly varying $L(1 / s)$. Hence $G(u)=\tilde{g}_{\alpha / 2}(b u)$ with suitable constant $b$.

Example. Let $X_{t}$ be a 1-dimensional standard Brownian motion and $f(x)$ be a bounded Borel function such that

$$
f \not \equiv 0 \text { a.e., } \quad \int\left|x^{3} f(x)\right| d x<\infty, \text { and } \int f(x) d x=0
$$

Since $G_{s}(x, y)=\frac{1}{\sqrt{2 s}} e^{-\sqrt{2 s}|x-y|}$ with $v(d x)=d x$, (B) (D) are satisfied if we set $h(s)=1 / \sqrt{2 s}, u(x, y)=-|x-y|, \varepsilon(x, y ; s)=\frac{1}{\sqrt{2 s}}\left(e^{-\sqrt{2 s}|x-y|}-1+\sqrt{2 s}|x-y|\right)$ and $\rho(x)=|x|+1$. To show that (A) is satisfied, we have only to notice that

$$
\left|G_{s}(x, y)-G_{s}(x, 0)\right| \leqq|y| .
$$

In fact,

$$
\begin{aligned}
\left|G_{s} f(x)\right| & =\left|\int G_{s}(x, y) f(y) d y\right|=\left|\int\left(G_{s}(x, y)-G_{s}(x, 0)\right) f(y) d y\right| \\
& \leqq \int|y f(y)| d y \quad(<\infty) .
\end{aligned}
$$


Hence we obtain the following;

$$
\lim _{t \rightarrow \infty} P_{x}\left\{\frac{1}{C_{1} t^{1 / 4}} \int_{0}^{t} f\left(X_{s}\right) d s<u\right\}=\tilde{g}_{1 / 4}(u), u \in \mathbb{R}^{1},
$$

where $\left(C_{1}\right)^{2}=-\frac{1}{\sqrt{2}} \iint|x-y| f(x) f(y) d x d y$.

Remark. The result in the example above can be obtained by another method. Let $f(x)(\not \equiv 0$ a.e.) be a bounded measurable function such that $x f(x)$ is summable and such that $\int f(x) d x=0$. Then

$$
G(x)=2 \int_{-\infty}^{x}(x-y) f(y) d y \text { and } F(x)=2 \int_{-\infty}^{x} f(y) d y
$$

are bounded functions. By Itô's formula, we obtain

$$
G\left(X_{t}\right)-G\left(X_{0}\right)=\int_{0}^{t} F\left(X_{s}\right) d X_{s}+\int_{0}^{t} f\left(X_{s}\right) d s
$$

Since $G(x)$ is bounded, we have only to show

$$
\lim _{t \rightarrow \infty} E_{x}\left\{\exp \frac{\lambda}{C_{1} t^{1 / 4}} \int_{0}^{t} F\left(X_{s}\right) d X_{s}\right\}=E_{1 / 2}\left(\lambda^{2}\right)
$$

where $\quad E_{1 / 2}\left(\lambda^{2}\right)=\sum_{n=1}^{\infty} \lambda^{2 n} / \Gamma(n / 2+1)=\int_{-\infty}^{\infty} e^{\lambda x} d \tilde{g}_{1 / 4}(x)$

$$
=\int_{0}^{\infty} e^{\lambda^{2} x} d g_{1 / 2}(x), \quad(\text { see Appendix })
$$

To prove this we use the Cameron-Martin formula;

$$
\begin{aligned}
& E_{x}\left\{\exp \frac{\lambda}{C_{1} t^{1 / 4}} \int_{0}^{t} F\left(X_{s}\right) d X_{s}\right\} \\
& =E_{x}\left\{\operatorname { e x p } \frac { \lambda ^ { 2 } } { 2 C _ { 1 } ^ { 2 } t ^ { 1 / 2 } } \int _ { 0 } ^ { t } F ( X _ { s } ) ^ { 2 } d s \cdot \operatorname { e x p } \left(\frac{\lambda}{C_{1} t^{1 / 4}} \int_{0}^{t} F\left(X_{s}\right) d X_{s}\right.\right. \\
& =E_{x}\left\{\exp \frac{\lambda^{2}}{2 C_{1}^{2} t^{1 / 2}} \int_{0}^{t} F\left(X_{s}^{t}\right)^{2} d s\right\}
\end{aligned}
$$

where $X^{t}$. is the solution of the following stochastic differential equation; 


$$
d X_{s}^{t}=d X_{s}+\frac{\lambda F\left(X_{s}^{t}\right)}{C_{1} t^{1 / 4}} d s
$$

Then using the method of C. Stone [5], we can prove

$$
\lim _{t \rightarrow \infty} E_{x}\left\{\exp \frac{\lambda^{2}}{2 C_{1}^{2} t^{1 / 2}} \int_{0}^{t} F\left(X_{s}^{t}\right)^{2} d s\right\}=E_{1 / 2}\left(\lambda^{2}\right) .
$$

This proves our assertion.

\section{§2. Auxialiary Results}

In order to prove the theorems in section 1 , we need some auxialiary results. Throughout this section we assume (A) (D). To simplify the notations, for any measurable function $u(x)$ defined on $E$, we denote $\sup _{x \in E}|u(x)| \mid \rho(x)$ by $\|u\|$.

Notice that $\lim _{n \rightarrow \infty}\left\|u_{n}\right\|=0$ is followed by $\lim _{n \rightarrow \infty} u_{n}(x)=0$ for each $x$.

Lemma 2.1. Let $g(x)=\int_{E} u(x, y) f(y) v(d y)$. Then,

(i) $\varlimsup_{s \rightarrow 0}\left\|G_{s} f(x)-g(x)\right\|=0$.

(ii) $g(x)$ is bounded on $E$.

(iii) $\varlimsup_{s \rightarrow 0}\left\|G_{s}(f g)-C h(s)\right\|<\infty$.

Proof. (i) follows immediately from (D.3), and (ii) from (A) and (i). By the definition of $g(x)$, we have,

$$
\begin{aligned}
& G_{s}(f g)(x)-C h(s) \\
& \quad=\int u(x, y) f(y) g(y) v(d y)+\int \varepsilon(x, y ; s) f(y) g(y) v(d y) .
\end{aligned}
$$

Since $g(x)$ is bounded, (D.2) and (D.3) imply (iii).

Q.E.D.

Lemma 2.2. For any $v_{s}(x), s>0\left(\varlimsup_{s \rightarrow 0}\left\|v_{s}\right\|<\infty\right)$,

(i) $\varlimsup_{s \rightarrow 0}\left\|\frac{G_{s}\left(f v_{s}\right)}{h(s)}\right\| \leqq K \varlimsup_{s \rightarrow 0}\left\|v_{s}\right\|$. 
(ii) $\varlimsup_{s \rightarrow 0}\left\|\frac{G_{s} f\left(G_{s}\left(f v_{s}\right)\right)}{h(s)}\right\| \leqq K \varlimsup_{s \rightarrow 0}\left\|v_{s}\right\|$

where $K$ is a positive constant which is independent of $v_{s}$.

Proof. Set $a(s)=\int f(y) v_{s}(y) v(d y)$, then we easily see that

$$
\varlimsup_{s \rightarrow 0}\left\|G_{s}\left(f v_{s}\right)(x)-a(s) h(s)\right\| \leqq K_{1} \varlimsup_{s \rightarrow 0}\left\|v_{s}\right\|
$$

where $K_{1}=\left\|\int|u(x, y) f(y)| \rho(y) v(d y)\right\|(<\infty)$ and that

$$
\varlimsup_{s \rightarrow 0}|a(s)| \leqq \int|f(y)| \rho(y) v(d y) \cdot \varlimsup_{s \rightarrow 0}\left\|v_{s}\right\| .
$$

Since $\|a(s)\| \leqq|a(s)|$, we obtain by (2.1) and (2.2) that

$$
\varlimsup_{s \rightarrow 0}\left\|\frac{G_{s}\left(f v_{s}\right)}{h(s)}\right\| \leqq K_{2} \varlimsup_{s \rightarrow 0}\left\|v_{s}\right\|
$$

where $K_{2}=\int|f(y)| \rho(y) v(d y) \quad(<\infty)$.

Thus (i) is proved, and furthermore, using (i) and (2.1), (2.2), we have,

$$
\begin{gathered}
\varlimsup_{s \rightarrow 0}\left\|\frac{G_{s}\left(f\left(G_{s}\left(f v_{s}\right)\right)\right)}{h(s)}\right\| \leqq \varlimsup_{s \rightarrow 0}\left\|\frac{G_{s} f\left(G_{s}\left(f v_{s}\right)-a(s) h(s)\right)}{h(s)}\right\|+\varlimsup_{s \rightarrow 0}|a(s)|\left\|G_{s} f\right\| \\
\leqq K_{2} \varlimsup_{s \rightarrow 0}\left\|G_{s}\left(f v_{s}\right)-a(s) h(s)\right\|+\varlimsup_{s \rightarrow 0} \sup _{x}\left|G_{s} f(x)\right| \varlimsup_{s \rightarrow 0}|a(s)| \\
\leqq K_{1} K_{2} \varlimsup_{s \rightarrow 0}\left\|v_{s}\right\|+K_{2} K_{3} \varlimsup_{s \rightarrow 0}\left\|v_{s}\right\|, \\
\text { where } K_{3}=\varlimsup_{s \rightarrow 0} \sup _{x}\left|G_{s} f(x)\right| .
\end{gathered}
$$

Lemma 2.3. Let $v_{1}(s, x)=G_{s} f(x)$ and $u_{1}(s, x)=\frac{G_{s}\left(f v_{1}\right)}{h(s)}$,

and

$$
\begin{aligned}
& u_{n+1}(s, x)=\frac{1}{h(s)}\left(G_{s} f\left(G_{s}\left(f u_{n}\right)\right)\right)(x), \\
& v_{n+1}(s, x)=\frac{1}{h(s)}\left(G_{s} f\left(G_{s}\left(f v_{n}\right)\right)\right)(x), n=1,2, \ldots
\end{aligned}
$$

Then, 
(i) $\varlimsup_{s \rightarrow 0}\left\|u_{n}(x)-C^{n}\right\|=0, \quad n=1,2, \ldots$

(ii) $\varlimsup_{s \rightarrow 0}\left\|v_{n}(x)\right\|<\infty, \quad n=1,2, \ldots$

Proof. We prove the assertion by induction. By Lemma 2.1

we have

$$
\begin{aligned}
& \varlimsup_{s \rightarrow 0}\left\|\frac{G_{s}(f g)}{h(s)}-C\right\|=0, \\
& \varlimsup_{s \rightarrow 0}\left\|G_{s} f-g\right\|=0 .
\end{aligned}
$$

Hence, using Lemma 2.2 (i),

$$
\begin{aligned}
\varlimsup_{s \rightarrow 0}\left\|u_{1}-C\right\| & \leqq \varlimsup_{s \rightarrow 0}\left\|\frac{G_{s}(f g)}{h(s)}-C\right\|+\varlimsup_{s \rightarrow 0}\left\|\frac{G_{s}\left(f\left(G_{s} f-g\right)\right)}{h(s)}\right\| \\
& =\varlimsup_{s \rightarrow 0}\left\|\frac{G_{s}\left(f\left(G_{s} f-g\right)\right)}{h(s)}\right\| \\
& \leqq K \varlimsup_{s \rightarrow 0}\left\|G_{s} f-g\right\|=0 .
\end{aligned}
$$

$\varlimsup_{s \rightarrow 0}\left\|v_{1}\right\|<\infty$ follows immediately from (A).

Next we assume (i) and (ii) are valid for $n$. Then using Lemma 2.2 (ii),

$$
\begin{gathered}
\varlimsup_{s \rightarrow 0}\left\|u_{n+1}-C^{n+1}\right\| \leqq \varlimsup_{s \rightarrow 0}\left\|\frac{G_{s} f\left(G_{s} f\left(u_{n}-C^{n}\right)\right)}{h(s)}\right\| \\
+\varlimsup_{s \rightarrow 0}\left\|C^{n}\left(\frac{G_{s} f G_{s} f}{h(s)}-C\right)\right\| \\
\leqq K \varlimsup_{s \rightarrow 0}\left\|u_{n}-C^{n}\right\|+|C|^{n} \varlimsup_{s \rightarrow 0}\left\|u_{1}-C\right\|=0,
\end{gathered}
$$

and

$$
\varlimsup_{s \rightarrow 0}\left\|v_{n+1}\right\| \leqq K \varlimsup_{s \rightarrow 0}\left\|v_{n}\right\|
$$

Now the induction is completed.

Q.E.D.

As an easy corollary of Lemma 2.3, we obtain the following;

Lemma 2.4. For each $x \in E$, 


$$
\begin{array}{r}
\lim _{s \rightarrow 0} \frac{1}{h(s)^{n / 2}} \overbrace{G_{s} f\left(G_{s} f\left(\cdots\left(G_{s} f\right) \cdots\right)\right)(x)=\frac{1+(-1)^{n}}{2}}^{n} \cdot C^{n}, \\
n=1,2, \ldots
\end{array}
$$

We assumed in this section $(A) \sim(D)$. However we remark that Lemma 2.4 is of course valid if we assume ( $\left.D^{\prime}\right)$ instead of (D). The proof turns out to be easier, so the details are omitted.

\section{§3. Proof of the Main Theorems}

Throughout this section we fix $x_{0} \in E$ and $E_{x_{0}}\{\cdot\}, P_{x_{0}}\{\cdot\}$ are denoted simply $E\{\cdot\}, P\{\cdot\}$ respectively. Now changing the variable we have

$$
\begin{aligned}
& s \int_{0}^{\infty} e^{-s t} E\left\{\left(\int_{0}^{t} f\left(X_{\tau}\right) d \tau\right)^{2}\right\} d t \\
& \quad=2 ! \int G_{s}\left(x_{0}, x_{1}\right) f\left(x_{1}\right) v\left(d x_{1}\right) \int G_{s}\left(x_{1}, x_{2}\right) f\left(x_{2}\right) v\left(d x_{2}\right) .
\end{aligned}
$$

Hence Lemma 2.4 provides us with

$$
\lim _{s \rightarrow 0} \frac{s}{h(s)} \int_{0}^{\infty} e^{-s t} E\left\{\left(\int_{0}^{t} f\left(X_{\tau}\right) d \tau\right)^{2}\right\} d t=2 C
$$

Notice that the left-hand side is of course nonnegative and consequently $C$ is nonnegative. (3.2) can be generalized easily as follows;

$$
\begin{gathered}
\lim _{s \rightarrow 0} \frac{s}{\{C h(s)\}^{n / 2}} \int_{0}^{\infty} e^{-s t} E\left\{\left(\int_{0}^{t} f\left(X_{\tau}\right) d \tau\right)^{n}\right\} d t=\frac{1+(-1)^{n}}{2} n ! \\
\text { (see [1]). }
\end{gathered}
$$

Since the integrand is not necessarily increasing, we cannot apply Tauberian theorem even if $h(s)$ varies regularly. So we have to make a detour if we want to evaluate the asymptotic behaviour of the moments of $\int_{0}^{t} f\left(X_{\tau}\right) d \tau$.

Let $T$ be a nonnegative random variable which is independent of $X$ such that $P\{T>x\}=c^{-x}$. Then we can rewrite (3.3) as follows; 


$$
\lim _{s \rightarrow 0} E\left\{\left(\frac{1}{\sqrt{C h(s)}} \int_{0}^{T / s} f\left(X_{\tau}\right) d \tau\right)^{n}\right\}=\frac{1+(-1)^{n}}{2} n !
$$

The right-hand side of (3.4) gives the $n$-th moment of the bilateral exponential distribution which belongs to the determinate case. Therefore (3.4) implies

$$
\lim _{s \rightarrow 0} P\left\{\frac{1}{\sqrt{C h(s)}} \int_{0}^{T / s} f\left(X_{\tau}\right) d \tau<x\right\}=\frac{1}{2} \int_{-\infty}^{x} e^{-|y|} d y
$$

Consequently,

$$
\lim _{s \rightarrow 0} P\left\{\left|\frac{1}{\sqrt{C h(s)}} \int_{0}^{T / s} f\left(X_{\tau}\right) d \tau\right|<x\right\}=1-e^{-x}, x>0 .
$$

We next introduce another process;

$$
M_{t}=g\left(X_{t}\right)+\int_{0}^{t} f\left(X_{\tau}\right) d \tau, \quad t \geqq 0,
$$

where $g(x)$ is the bounded function defined in Lemma 2.1. Taking in mind that $G_{s} f(x) \rightarrow g(x)$ (the convergence being dominated by a positive constant), we see that $M_{t}$ is a martingale. Since $g(x)$ is bounded, (3.5) and (3.6) provide us with

$$
\lim _{s \rightarrow 0} P\left\{\frac{1}{\sqrt{C h(s)}} M_{T / s}<x\right\}=\frac{1}{2} \int_{-\infty}^{x} e^{-|y|} d y,
$$

and

$$
\lim _{s \rightarrow 0} P\left\{\frac{1}{\sqrt{C h(s)}}\left|M_{T / s}\right|<x\right\}=1-e^{-x}
$$

It also follows from (3.4) that the moment of arbitrary order of $\frac{1}{\sqrt{C h(s)}} M_{T / s}$ is bounded. Hence we obtain,

$$
\begin{aligned}
& \lim _{s \rightarrow 0} E\left\{\left(\frac{1}{\sqrt{C h(s)}} M_{T / s}\right)^{n}\right\}=\frac{1+(-1)^{n}}{2} n ! \\
& \lim _{s \rightarrow 0} E\left\{\left|\frac{1}{\sqrt{C h(s)}} M_{T / s}\right|^{n}\right\}=n !
\end{aligned}
$$

or equivalently, 


$$
\begin{aligned}
& \lim _{s \rightarrow 0} \int_{0}^{\infty} e^{-t} E\left\{\left(\frac{1}{\sqrt{C h(s)}} M_{t / s}\right)^{n}\right\} d t=\frac{1+(-1)^{n}}{2} n ! \\
& \lim _{s \rightarrow 0} \int_{0}^{\infty} e^{-t} E\left\{\left|\frac{1}{\sqrt{C h(s)}} M_{t / s}\right|^{n}\right\} d t=n !
\end{aligned}
$$

Changing the variables, we have,

$$
\begin{aligned}
& \lim _{s \rightarrow 0} \frac{s}{\{C h(s)\}^{n / 2}} \int_{0}^{\infty} e^{-s t} E\left(M_{t}^{n}\right) d t=\frac{1+(-1)^{n}}{2} n !, \\
& \lim _{s \rightarrow 0} \frac{s}{\{C h(s)\}^{n / 2}} \int_{0}^{\infty} e^{-s t} E\left(\left|M_{t}\right|^{n}\right) d t=n !, n=1,2, \ldots .
\end{aligned}
$$

Consequently we also have,

$$
\lim _{s \rightarrow 0} \frac{s}{\{C h(s)\}^{n / 2}} \int_{0}^{\infty} e^{-s t} E\left\{\left|M_{t}\right|^{n}+M_{t}^{n}\right\} d t=\frac{3+(-1)^{n}}{2} n !
$$

Since $M_{t}$ is a martingale, both $E\left\{\left|M_{t}\right|^{n}\right\}$ and $E\left\{\left|M_{t}\right|^{n}+M_{t}^{n}\right\}$ are nondecreasing in $t(n=1,2, \ldots)$. Hence, if $h(s)=s^{-\alpha} L(1 / s)$, we can apply the Karamata's Tauberian theorem. By (3.11) and (3.12) we obtain,

$$
\begin{aligned}
& \lim _{t \rightarrow \infty} \frac{1}{\left\{C h\left(\frac{1}{t}\right)\right\}^{n / 2}} E\left\{\left|M_{t}\right|^{n}\right\}=n ! / \Gamma\left(\frac{\alpha n}{2}+1\right) \\
& \lim _{t \rightarrow \infty} \frac{1}{\left\{C h\left(\frac{1}{t}\right)\right\}^{n / 2}} E\left\{\left|M_{t}\right|^{n}+M_{t}^{n}\right\}=\frac{3+(-1)^{n}}{2} n ! / \Gamma\left(\frac{\alpha n}{2}+1\right),
\end{aligned}
$$

and consequently we have,

$$
\lim _{t \rightarrow \infty} \frac{1}{\left\{C h\left(\frac{1}{t}\right)\right\}^{n / 2}} E\left\{M_{t}^{n}\right\}=\frac{1+(-1)^{n}}{2} n ! / \Gamma\left(\frac{\alpha n}{2}+1\right), \quad n=1,2 \ldots
$$

The right-hand side of (3.15) is the $n$-th moment of the bilateral Mittag-Leffler distribution, which belongs to the determinate case. $0 \leqq$ $\alpha \leqq 1$ is rather trivial by Lemma 2.1 (iii). Thus the proof of Theorem 1 is completed.

We next prove Theorem 2. We need little modification to the proof of Theorem 2 in [1]. Since $u(t)$ is nondecreasing, we can choose a 
nondecreasing function $\phi(t)$ with values in $[0, \infty]$ and a sequence $s_{n} \rightarrow 0$, so that

$$
\frac{1}{\sqrt{C h\left(s_{n}\right)}} u\left(t / s_{n}\right) \longrightarrow \phi(t), \quad n \longrightarrow \infty
$$

at each continuity point of $\phi(t)$. Then,

$$
\begin{aligned}
& \lim _{n \rightarrow \infty} \int_{0}^{\infty} e^{-t} P\left\{\frac{1}{\sqrt{C h\left(s_{n}\right)}}\left|\int_{0}^{t / s_{n}} f\left(X_{\tau}\right) d \tau\right|<x\right\} d t \\
& \quad=\lim _{n \rightarrow \infty} \int_{0}^{\infty} e^{-t} P\left\{\frac{u\left(t / s_{n}\right)}{\sqrt{C h\left(s_{n}\right)}} \frac{1}{u\left(t / s_{n}\right)}\left|\int_{0}^{t / s_{n}} f\left(X_{\tau}\right) d \tau\right|<x\right\} d t \\
& \quad=\int_{0}^{\infty} e^{-t} \widetilde{G}(x / \phi(t)) d t, \quad \text { a.e. } \quad x>0,
\end{aligned}
$$

with trivial conventions; $\widetilde{G}\left(\frac{x}{0}\right)=1$ and $\widetilde{G}\left(\frac{x}{\infty}\right)=\widetilde{G}(0)$ where $\widetilde{G}(x)=G(x+0)$ $-G(-x-0), x \geqq 0$.

Now (3.6) and (3.16) provides us with

$$
\int_{0}^{\infty} e^{-t} \tilde{G}(x / \phi(t)) d t=1-e^{-x}
$$

letting $x \rightarrow \infty$, we have $\tilde{G}(x / \phi(t)) \rightarrow 1$, a.e.t. Since $\tilde{G}(0)<1$ by the assumption, we obtain $\phi(t)<\infty, t>0$. Similarly we also have $\phi(t)>0, t>0$. Darling and $\mathrm{Kac}[1]$ proved that (3.17) determines $\phi(t)$ uniquely, which implies

$$
\lim _{s \rightarrow 0} \frac{u(t / s)}{\sqrt{C h(s)}}=\phi(t)
$$

This proves that $h(s)$ varies regularly for some exponent $\alpha$. Since $0 \leqq \alpha \leqq 1$ is rather trivial as in the proof of Theorem 1 , our assertion is now proved.

\section{§4. Limit Theorems for Symmetric Stable Processes}

Let $X_{t}$ be an additive process on $\boldsymbol{R}^{n}(n=1,2)$ such that $E_{0}\left\{e^{i<\xi, X_{t}>}\right\}$ $=e^{-t|\xi|^{\alpha}}$. We assume that $X_{t}$ is recurrent; i.e. $1 \leqq \alpha \leqq 2$ if $n=1$, and $\alpha=2$ if $n=2$. Green kernel $G(x, y)$ (with respect to Lebesgue measure) 
has the following representation;

$$
G_{s}(x, y)= \begin{cases}\frac{1}{\pi} \int_{0}^{\infty} \frac{\cos (x-y) \xi}{s+\xi^{\alpha}} d \xi & \text { if } n=1,1 \leqq \alpha \leqq 2 \\ \frac{1}{2 \pi} \int_{0}^{\infty} \frac{\cos |x-y| \xi}{\sqrt{s+\xi^{2}}} d \xi & \text { if } n=2, \alpha=2\end{cases}
$$

Therefore we have,

$$
G_{s}(x, y)=h(s)+u(x, y)+\varepsilon(x, y ; s)
$$

where

$$
\begin{aligned}
& h(s)= \begin{cases}\frac{1}{\alpha \sin (\pi / \alpha)} s^{1 / \alpha-1} & \text { if } n=1<\alpha \leqq 2 \\
\frac{1}{\pi} \log (1 / s) & \text { if } n=\alpha=1 \\
\frac{1}{4 \pi} \log (4 / s)+\gamma / 2 & \text { if } n=\alpha=2\end{cases} \\
& u(x, y)= \begin{cases}\frac{1}{2 \cos \left(\left.\frac{1}{\pi} \log \Gamma(\alpha) \frac{1}{|x-y|}\right|^{1}-\alpha\right.} & \text { if } n=1<\alpha \leqq 2 \\
\frac{1}{2 \pi} \log \frac{1}{|x-y|} & \text { if } n=\alpha=1\end{cases}
\end{aligned}
$$

and $\varepsilon(x, y ; s)$ converges to 0 uniformly on each compact set in $\boldsymbol{R}^{1} \times$ $\boldsymbol{R}^{1}$ or $\boldsymbol{R}^{2} \times \boldsymbol{R}^{2}$. Let $f(x)$ be a bounded Borel function with compact support such that $\int f(x) d x=0$. Using a similar argument which we used in the example in section 1, we see that the assumptions (A) $\left(D^{\prime}\right)$ are satisfied. Hence we obtain

\section{Theorem 3.}

$$
\lim _{t \rightarrow \infty} P\left\{\frac{1}{\sqrt{C h(1 / t)}} \int_{0}^{t} f\left(X_{\mathrm{s}}\right) d s<u\right\}=\tilde{g}_{\beta / 2}(u)
$$

where $\beta= \begin{cases}1-1 / \alpha & \text { if } n=1 \leqq \alpha \leqq 2 \\ 0 & \text { if } n=\alpha=2\end{cases}$ 
and $\quad C=\iint u(x, y) f(x) f(y) d x d y$.

\section{§5. Limit Theorems for 1-Dimensional Diffusion Processes}

Let $m(d x)$ be a nonnegative Radon measure on $\mathbb{R}^{1}$. Then we can obtain a generalized diffusion process $X_{t}$ with the infinitesimal generator $\frac{d}{d m} \frac{d}{d x}$ from 1-dimensional Brownian motion by means of time change (see [5]). If the support of $m(d x)$ is an interval $Q$, then $X_{t}$ becomes a diffusion process on $Q$ with reflecting barrier when the boundary is finite.

Remark that $X_{t}$ is a recurrent, conservative Markov process on $E=\operatorname{supp} m(d x) .{ }^{1)}$

Now let $\{\phi(x, \lambda), \psi(x, \lambda)\}$ be the system of the solutions of the following equations.

$$
\begin{aligned}
& \phi(x, \lambda)=1-\lambda \int_{x_{1}}^{x}(x-y) \phi(y, \lambda) m(d y) \\
& \psi(x, \lambda)=x-\lambda \int_{x_{1}}^{x}(x-y) \psi(y, \lambda) m(d y)-x_{1}, \quad-\infty<x<\infty .
\end{aligned}
$$

where $\int_{y}^{x}=\int_{[y, x)}$ if $y<x$, and $=-\int_{[x, y)}$ if $x<y$.

Then it is well known that the following hold for each $s>0$.

$$
\begin{aligned}
1 \leqq & \phi(x,-s) \leqq e^{s \sigma(x)} \\
& |\psi(x,-s)| \leqq\left|x-x_{1}\right| e^{s \sigma(x)}
\end{aligned}
$$

where $\sigma(x)=\int_{x_{1}}^{x}(x-\xi) m(d \xi)$.

We next define $h_{i}(s), i=1,2$.

$$
h_{i}(s)=\lim _{x \rightarrow(-1)^{i} \infty}(-1)^{i} \frac{\psi(x,-s)}{\phi(x,-s)}, s>0, i=1,2 .
$$

Then $u_{i}(x, s)=\phi(x,-s)-(-1)^{i} \psi(x,-s) / h_{i}(s), i=1,2$ are positive solutions of $\left(\frac{d}{d m} \frac{d}{d x}-s\right) u=0 ; u_{1}(\cdot, s)$ is nondecreasing and $u_{2}(\cdot, s)$ nonincreasing.

1) We assume that $E$ contains at least two points, say $x_{1}, x_{2},\left(x_{1}>x_{2}\right)$. 
Green kernel of $X_{t}$ with respect to $m(d x)$ is given by the following;

$$
G_{s}(x, y)=\left\{\begin{array}{lll}
h(s) u_{1}(x, s) u_{2}(y, s) & \text { if } & x \leqq y \\
h(s) u_{2}(x, s) u_{1}(y, s) & \text { if } & y<x
\end{array}\right.
$$

where $h(s)=\left(\frac{1}{h_{1}(s)}+\frac{1}{h_{2}(s)}\right)^{-1}$.

Notice that $\lim _{s \rightarrow 0} \operatorname{sh}(s)=m(-\infty, \infty)^{-1}$.

\section{Lemma 5.1.}

$$
\left|G_{s}(x, y)-G_{s}\left(x, x_{1}\right)\right| \leqq 2\{s h(s) \sigma(y)+|y|\} e^{2 s \sigma(y)} \quad s>0, x, y \in \boldsymbol{R}^{1}
$$

Proof. Since we have the explicit representation of $G_{s}(x, y)$, it is not difficult to prove the assertion.

Q.E.D.

By Lemma 5.1, it is easy to see that the assumption (A) is satisfied if $f(x)$ fulfils the following;

(A') $f(x)$ is a bounded Borel function with compact support such that $\int f(x) m(d x)=0$ but $f(x) \not \equiv$ m-a.e.

In order to prove $\left(D^{\prime}\right)$ is satisfied, we need further assumption.

$\left(\mathrm{D}^{\prime \prime}\right)$ The limit $\theta=\lim _{s \rightarrow 0} h(s) / h_{1}(s)$ exists.

Remark that $\left(\mathrm{D}^{\prime \prime}\right)$ is valid whenever $m\left(-\infty, x_{1}\right)<\infty$. In fact, $\theta=$ $m\left(-\infty, x_{1}\right) / m(-\infty, \infty)$.

\section{Lemma 5.2. Let}

$$
u(x, y)=-(x \vee y)-\theta \cdot(x+y)+(\sigma(x)+\sigma(y)) / m(-\infty, \infty)
$$

Then $\varepsilon(x, y ; s)=G_{s}(x, y)-h(s)-u(x, y) \rightarrow 0, s \rightarrow 0$, the convergence being uniform on each compact set of $\boldsymbol{R}^{1} \times \boldsymbol{R}^{1}$.

This result is obtained with $H$. Watanabe and will be published elsewhere.

With $h(s)$ and $u(x, y)$ given above, we can see that $(\mathrm{B}) \sim\left(\mathrm{D}^{\prime}\right)$ are satisfied.

Finally we need conditions for the regular variation of $h(s)$. But using the results in [4], we easily obtain that if $m(d x)$ satisfies the 
following condition (R), then $h(s)$ varies regularly at 0 with exponent $-\alpha$.

(R) $m(-x, x)$ varies regularly at $\infty$ with exponent $1 / \alpha-1(0 \leqq \alpha \leqq 1)$ and satisfies one of the following conditions.

(R.1) $m[0, x) \sim \operatorname{cm}(-x, 0)$ as $x \rightarrow \infty$, with some positive constant $c$.

(R.2) $\lim _{x \rightarrow \infty} \frac{m(-\lambda x, 0)}{m[0, x)}=0[$ or $\infty]$ for each $\lambda>0$.

We remark that if $(R)$ is satisfied then $\left(D^{\prime \prime}\right)$ is also satisfied. Therefore we obtain the following;

Theorem 4. If $\left(\mathrm{A}^{\prime}\right)$ and $(\mathrm{R})$ are satisfied, then for each $x \in E$

$$
\lim _{t \rightarrow \infty} P_{x}\left\{\frac{1}{\sqrt{C h(1 / t)}} \int_{0}^{t} f\left(X_{s}\right) d s<u\right\}=\tilde{g}_{\alpha / 2}(u)
$$

where $C=-\frac{1}{2} \iint|x-y| f(x) f(y) m(d x) m(d y)$.

\section{Example.}

If $m[0, x) \sim x^{\beta}$ as $x \rightarrow \infty$ and $m(-x, 0) \sim x^{\gamma} c^{x}$ as $x \rightarrow \infty$ for some nonnegative constants $\beta$ and $\gamma$, then

$$
\begin{aligned}
& h_{1}(s) \sim \log (1 / s) \quad(s \downarrow 0) \\
& h_{2}(s) \sim \operatorname{const} \cdot s^{-1 /(1+\beta)} \quad(s \downarrow 0)
\end{aligned}
$$

and consequently,

$$
h(s) \sim \log (1 / s) \quad(s \downarrow 0) .
$$

Therefore we obtain

$$
\lim _{t \rightarrow \infty} P_{x}\left\{\frac{1}{\sqrt{C \log t}} \int_{0}^{t} f\left(X_{s}\right) d s<u\right\}=\frac{1}{2} \int_{-\infty}^{u} e^{-|y|} d y
$$

where $\quad C=-\frac{1}{2} \iint|x-y| f(x) f(y) m(d x) m(d y)$.

In case $m(-\infty, 0)=0$, matters become clearer. As an easy corollary of Theorem 4 in [4], we have the following; 
Theorem 5. Suppose $\left(\mathrm{A}^{\prime}\right)$ is satisfied. If $m[0, x)$ varies regularly' at $\infty$ with exponent $\beta(0 \leqq \beta \leqq \infty)$ then for each $x \in E$,

$$
\lim _{t \rightarrow \infty} P_{x}\left\{\frac{1}{\sqrt{C h(1 / t)}} \int_{0}^{t} f\left(x_{s}\right) d s<u\right\}=\tilde{g}_{1 /(2(\beta+1))}(u) .
$$

Theorem 6. Suppose $\left(\mathrm{A}^{\prime}\right)$ is satisfied. If there exists a nondegenerate distribution function $G(u)$ such that

$$
\lim _{t \rightarrow \infty} P_{x}\left\{\frac{1}{u(t)} \int_{0}^{t} f\left(X_{s}\right) d s<u\right\}=G(u) \quad \text { a.e. } u
$$

holds for some nondecreasing function $u(t) \uparrow \infty$, then $m[0, x)$ varies regularly at $\infty$ with some exponent $\beta(0 \leqq \beta \leqq \infty)$. Hence $G(u)=$ $\tilde{g}_{\frac{1}{2}(\beta+1)^{-1}}(b u)$ with appropriate constant $b$.

\section{Appendix}

The distribution function of Mittag-Leffler distribution of order $\alpha(0 \leqq \alpha<1)$ is given by

$$
g_{\alpha}(x)=\frac{1}{\pi \alpha} \int_{0}^{x} \sum_{j=1}^{\infty} \frac{(-1)^{j-1}}{j !} \sin \pi \alpha j \Gamma(\alpha j+1) y^{j-1} d y, x>0
$$

and the moments of this distribution are given by $k ! / \Gamma(\alpha k+1), k=0$, $1,2, \ldots$, which belongs to the determinate case.

Bilateral Mittag-Leffler distribution of order $\alpha(0 \leqq \alpha<1)$ is the distribution the moment of which is $\frac{1+(-1)^{k}}{2} \frac{k !}{\Gamma(\alpha k+1)}$, which also belongs to the determinate case. Hence it is easy to see that the distribution function of bilateral Mittag-Leffler distribution is given by

$$
\tilde{g}_{\alpha}(x)=\frac{1}{2 \pi \alpha} \int_{-\infty}^{x} \sum_{j=1}^{\infty} \frac{(-1)^{j-1}}{j !} \sin \pi \alpha j \Gamma(\alpha j+1)|y|^{j-1} d y .
$$

We remark that for the special case of $\alpha=1 / 2$ we obtain

$$
\tilde{g}_{1 / 2}(x)=\frac{1}{2 \sqrt{\pi}} \int_{-\infty}^{x} e^{-y^{2} / 4} d y
$$

and of $\alpha=0$, 


$$
\tilde{g}_{0}(x)=\frac{1}{2} \int_{-\infty}^{x} e^{-|y|} d y
$$

\section{References}

[1] Darling, D. A., and Kac, M., On occupation times for Markov processes, Trans. Amer. Math. Soc., 84 (1957), 444-458.

[2] Dobrusin, R. L., Two limit theorems for the simplest random walk on a line, Uspehi Math. Nauk, 10 (1955), 139-146.

[ 3 ] Itô, K., and McKean, H. P., Diffusion processes and their sample paths, Springer, Berlin-Heidelberg-New York (1955).

[4] Kasahara, Y., Spectral theory of generalized second order differential operators and its applications to Markov processes, Japan. J. Math., 1 (1975), 67-84.

[5] Stone, C., Limit theorems for random walks, birth and death processes, and diffusion processes, Illinois J. Math., 7 (1963), 638-660.

[6] Tanaka, H., Certain limit theorems concerning one-dimensional diffusion processes, Mem. Fac. Sci. Kyushu Univ., 12 (1958), 1-11. 